\title{
Rapid Assessment of Soil Quality Indices Using Infrared Reflectance Spectroscopy
}

\author{
Darusman $^{1}$, Zulfahrizal $^{2}$, Agus Arip Munawar ${ }^{3}$ \\ ${ }^{1}$ Department of Soil Science, Universitas Syiah Kuala, Banda Aceh - Indonesia \\ ${ }^{2,3}$ Department of Agricultural Engineering, Universitas Syiah Kuala, Banda Aceh - Indonesia
}

$\{3$ aamunawar@unsyiah.ac.id $\}$

\begin{abstract}
In this present study, we investigated the use of near infrared reflectance spectroscopy (NIRS) as a rapid and robust method to assess and evaluate soil quality namely soil carbon organic (SOC) and $\mathrm{pH}$. Diffuse reflectance spectral data were acquired and recorded for $20 \mathrm{~g}$ soil samples from four different site locations in Aceh province. Spectra data, in the wavelength range of 1000-2500 $\mathrm{nm}$, were corrected and enhanced using de-trending (DT). Actual SOC and $\mathrm{pH}$ parameters were measured using standard laboratory procedures whilst prediction models, used to predict SOC and $\mathrm{pH}$ of soil samples, were established using integration principal component analysis and multiple linear regression (PCA+MLR) approach. Prediction performances were evaluated and justified based on statistical indicators: coefficient correlation (r), root mean square error (RMSE) and residual predictive deviation (RPD) index. The results showed that both soil quality indices ( $\mathrm{SOC}$ and $\mathrm{pH}$ ) can be predicted simultaneously with coefficient maximum correlation (r) were 0.97 for $\mathrm{SOC}$ and 0.99 for $\mathrm{pH}$, whereas residual predictive deviation (RPD) indexed were 4.84 for $\mathrm{pH}$ and 3.96 for SOC respectively. It may conclude that near-infrared reflectance spectroscopy can be used as a fast, robust and accurate method in assessing soil quality indices in the form of soil carbon organic (SOC) and $\mathrm{pH}$ simultaneously.
\end{abstract}

Keywords: NIRS, Soil, SOC, Carbon-Organic, pH

\section{Introduction}

To grow optimally, plants require healthy soil condition whether it is food crops or plantations. In fact, the soil must possess physical and chemical properties that are suitable for plant growth. Healthy soil conditions can be seen generally from its texture, structure, and humidity. Soil chemical properties are related to the number of nutrients needed by plants, with the amount needed will be different for each growing phase [1]. Soil fertility is largely determined by the presence of nutrients in the soil, both macronutrients, secondary, and micronutrients. Nutrients that are lacking in the soil will obviously affect plant growth. The growth rate will be very disturbed and susceptible to disease because soil nutrients do not meet the minimum requirements needed. Conversely, the number of excessive nutrients in the soil will also affect plant growth and the surrounding environment [2], [3].

In precision agriculture practice, fertilization and excessive use of fertilizer will cause pollution to the environment because it can cause artificial nutrient deposits that are not utilized by plants. This, of course, will accumulate and make new problems especially pollution due to the use of pesticides, fertilizers, and fertilizers that are above the normal amount [3]. 
Carbon organic or sometimes called as organic carbon content (SOC) and pH, are two of the main soil characteristics that play an important role in precision agriculture practice. The amount of SOC and $\mathrm{pH}$ must be known the certainty and precisely because they will determine the fertilization dose and the use of the right fertilizer so that the optimum amount of nutrients in the soil is sufficient [1], [2]. Organic carbon levels and $\mathrm{pH}$ play an important role in agricultural practices because these factors are able to improve the availability of nutrients and soil texture. Thus, knowing these two factors (organic carbon and $\mathrm{pH}$ ) will help in the decision-making process in precision agriculture or precision farming practices, especially for the selection of plants that are suitable for the existing environmental conditions. In addition, this will also determine suitable types of fertilization and irrigation to produce maximum agricultural production.

It is difficult to predict organic carbon (SOC) content and $\mathrm{pH}$ rapidly and in real time. This requires testing in a laboratory which is normally time-consuming, while the soil conditions must be immediately known so that plants can grow optimally and ideally. In addition, conventional testing in the laboratory is also sometimes complicated in the process, requiring chemicals and can cause environmental pollution [4].

Alternative fast, robust, non-destructive and pollution free methods are required to determine quality parameters of sol carbon organics and other soil characteristics. Near-infrared reflectance spectroscopy (NIRS) has been developed and widely applied in many sectors including in agriculture and soil science [5].

Near-infrared reflectance spectroscopy (NIRS) technique works based on the principle of interaction of electromagnetic radiation with biological objects like soil. This method is potential to be applied to determine soil nutrients and other related properties including carbon organic and $\mathrm{pH}$. The advantage of this method is: simple sample preparation, non-destructive in nature, no chemical waste, pollution free, simultaneous and high speed of analysis. [5].

The overall research findings of numerous studies on the application of near-infrared spectroscopy (NIRS), shows that NIRS was feasible to be applied as a rapid and non-destructive tool for quality attributes prediction in agricultural sectors. The prediction model performance was sufficiently robust and accurate with correlation coefficient (r) range of $0.93-0.99$ and residual predictive deviation (RPD) index was $1.53-4.68$ which is categorized as coarse, sufficient and excellent prediction models respectively.

Based on advantages and excellence of NIRS as a new, fast, simultaneous and robust method to determine agricultural and food qualities, we attempted to apply the NIRS method in predicting soil quality parameter in form of $\mathrm{C}$-organic and $\mathrm{pH}$. Prediction models were established using soil spectra data using a combination between principal component analysis and multiple linear regression (PCA+MLR) method. Obtained results were then compared to actual SOC and $\mathrm{pH}$ measured by standard laboratory procedures.

\section{Method}

\subsection{Samples}

A bulk of soil samples from four different site locations in Banda Aceh and Aceh Besar area were taken and stored for two days to equilibrate before spectra acquisition and further chemical analysis. 


\subsection{Near-infrared spectrum acquisition}

Infrared spectral data in the form of diffuse reflectance spectrum were taken of all soil samples using an infrared instrument (FTIR, Thermo Nicolet Antaris II MDS). The basic measurement with the probe detector was chosen as a basic measurement in high-resolution format. Infrared spectrum was collected and recorded in wavenumbers range from 4000 to 10 $000 \mathrm{~cm}^{-1}$ with co-added 32 scans and averaged. Spectra data were stored in a local computer with three different file formats (*.SPA, *.JDX and *.CSV) [5].

\subsection{C-organic (SOC) and $\mathrm{pH}$ measurement}

Once after spectra collection was completed, all soil samples were taken immediately to measure soil organic carbon (SOC) and $\mathrm{pH}$ using elemental analyzer and thermal conductivity detector. Soil organic carbon was expressed as \% SOC [1]. Actual carbon organic and $\mathrm{pH}$ data were measured in triplicate and averaged.

\subsection{Spectra data correction}

In order to obtain accurate and robust prediction results, infrared spectra data were enhanced and corrected using the de-trending (DT) method [5].

\subsection{C-organic and $\mathrm{pH}$ prediction model}

Prediction models used to predict SOC or C-organic of soil samples were established and developed using original untreated spectra data (defined as a raw spectrum) and enhanced spectra data (DT). Prediction models were established using combination between principal component analysis and multiple linear regression (PCA+MLR) [5], [6].

\subsection{Model evaluation performance}

Soil organic prediction performances were evaluated for their accuracies and robustness using several statistical indicators: coefficient of determination $\left(\mathrm{R}^{2}\right)$, correlation coefficient ( $\left.\mathrm{r}\right)$, root mean square error (RMSE) and the residual predictive deviation (RPD) (Munawar et al, 2016; Munawar et al, 2013). It is obvious that the good model should have high $\mathrm{R}^{2}$ and $\mathrm{r}$ coefficient, the low value of RMSE and few number latent variables of PCA [7], [8].

\section{Result and Discussion}

\subsection{Typical spectra of soil sample}

Typical diffuse reflectance spectrum for soil samples was presented in Fig.1. it shows several peaks represent the vibration of molecular bonds of C-C, O-H, N-H, C-H-O, and C-H. Original spectra data before correction were still interference due to noise resulted from light scattering [9], [10]. 


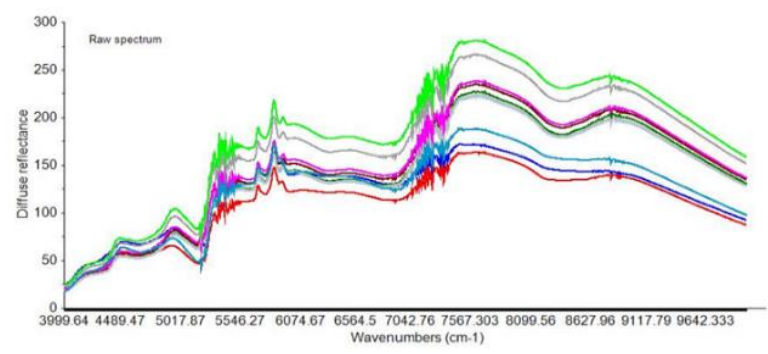

Fig.1. Typical diffuse reflectance spectra data of soil samples before correction.

These noises were corrected using pre-treatment method namely de-trending (DT). There are still many correction methods such as multiplicative scatter correction (MSC), mean normalization (MN), standard normal variate (SNV), Mean centering (MC) and combination among them. As shown in Fig. 2, DT correction method clearly enhance spectra appearance and remove some noises due to light scattering.

Spectral data acquired from the near infrared instrument generally contain background information and noises which are interfered and affected desired relevant soil quality information such as $\mathrm{C}$-organic and $\mathrm{pH}$. Interfering spectral parameters, such as light scattering, path length variations, and random noise resulted from variable physical sample properties or instrumental effects need to be removed or minimized in order to obtain accurate, robust and stable calibration models. Thus, it is very necessary to pre-process spectral data prior to prediction model development used to predict $\mathrm{C}$-organic.

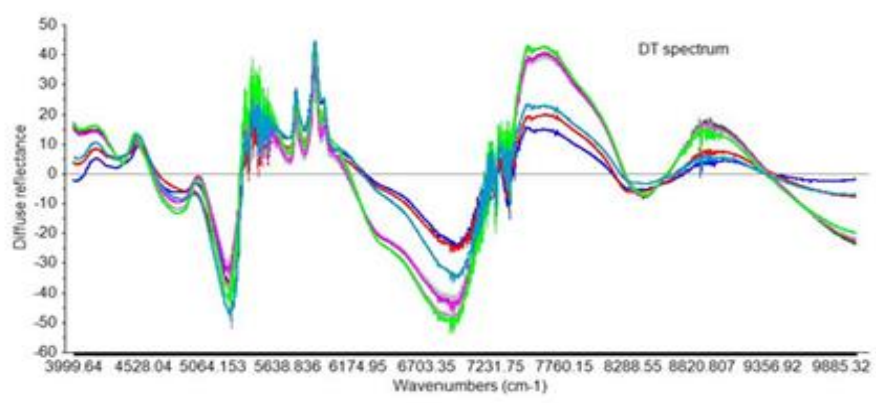

Fig.2. Diffuse reflectance spectra after corrected and enhanced using the de-trending (DT) correction method

As shown in Fig.2, spectra data were corrected and enhanced using DT, MSC, and combination of DT+MSC. The de-trending pre-treatment method tends to remove nonlinear trends in spectroscopic data, while MSC method used to reduce amplification due to light scattering and offset due to additive chemical effects in near-infrared spectra.

\subsection{Prediction models}

After spectra pre-processing were completed, we established prediction models used to predict C-organic on soil samples. Partial least squares regression (PLSR) models were built 
based on untreated and treated spectra using soil samples datasets with the wavenumbers range from $4000-10000 \mathrm{~cm}^{-1}$. The correlation coefficient (r), standard error prediction (RMSE) and residual predictive deviation (RPD) index, were compared.

$\mathrm{C}$-organic prediction model consists of the relationship between the observed response variable $\mathrm{y}$ (Y-variables: soil $\mathrm{C}$-organic) and the independent variable $\mathrm{x}$ (X-variables: diffuse near-infrared reflectance spectrum). The primary information that can be gathered from the interaction of the near-infrared radiation with the biological object is its physical, optical and chemical properties. Prediction result for soil C-organic (SOC) was shown in Table 1.

Table 1. SOC prediction performance using diffuse reflectance spectra data

\begin{tabular}{ccccc}
\hline Spectrum & \multicolumn{4}{c}{ Statistical indicator } \\
& $\mathrm{R}^{2}$ & $\mathrm{r}$ & RMSE & $\mathrm{RPD}$ \\
\hline \hline Raw & 0.81 & 0.90 & 0.23 & 3.14 \\
DT & 0.95 & 0.97 & 0.12 & 3.96
\end{tabular}

$R^{2}$ : coefficient of determination, $r$ : correlation coefficient, RMSE: root means square error, RPD: residual predictive deviation, DT: de-trending..

Firstly, C-organic (SOC) prediction model was developed using untreated raw spectral data. It achieved correlation coefficient of 0.90 , with $\mathrm{RMSE}$ value $=0.23$ and $\mathrm{RPD}$ index $=3.14$. When the prediction model was developed using DT spectra data, the correlation coefficient was increased become 0.97 and RMSE error prediction was decreased to 0.12 and RPD index obviously better than previous. Moreover, accuracy and robustness of C-organic prediction model were significantly improved when the prediction model was developed using DT spectra data. It achieved $r=0.97$ with $\mathrm{RPD}=3.96$ and error decreased to 0.12. Scatter plot derived from actual C-organic versus predicted C-organic were presented in Figure 3.
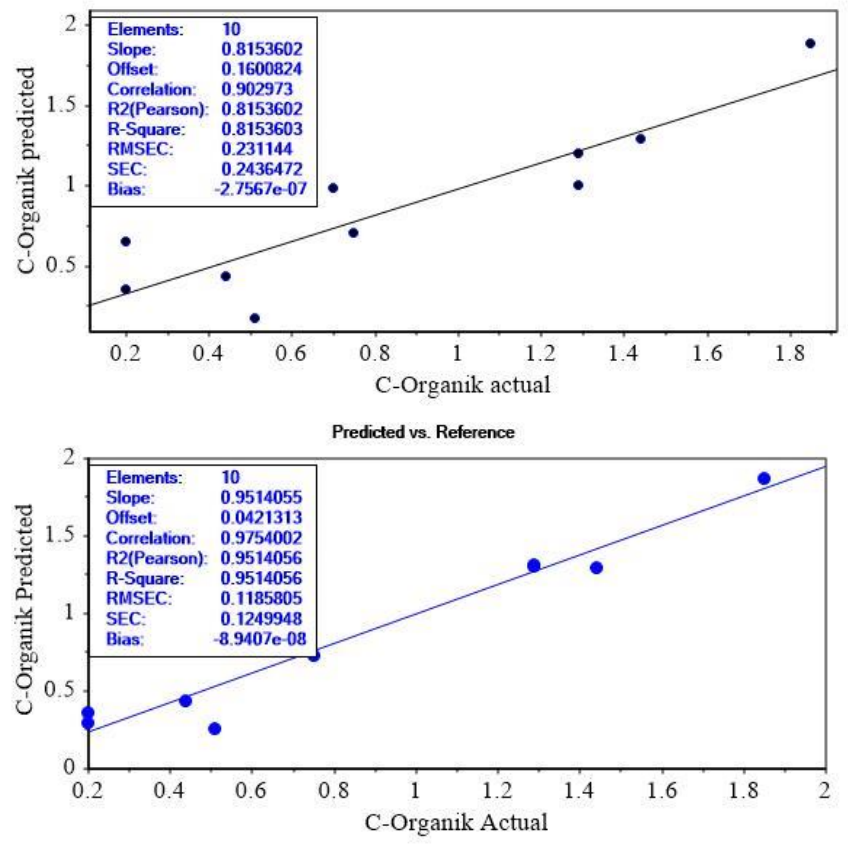

Fig. 3. Scatter plot between actual and predicted SOC of soil samples using NIR spectra data. 
Furthermore, the $\mathrm{pH}$ of soil samples was also predicted using the same spectra data as SOC prediction. The prediction model was established using raw untreated and DT treated spectrum by regressing $\mathrm{pH}$ actual data as $\mathrm{Y}$ variable and spectra data as $\mathrm{X}$ variable. The models were constructed using PCA+MLR method and the result is presented in Table 2. As shown in Table 2 , the NIRS method can predict $\mathrm{pH}$ even using raw spectrum data. The correlation coefficient is 0.94 , with RMSE value $=0.07$ and RPD index $=4.28$. Just like in SOC prediction, de-trending (DT) correction method can improve prediction accuracy and robustness. It achieved maximum correlation coefficient $r=0.99$ and RPD index $=4.84$ with lowest error $\mathrm{RMSE}=0.03$. Scatter plot derived from actual $\mathrm{pH}$ versus predicted $\mathrm{pH}$ were presented in Figure 4.

Table 2. $\mathrm{pH}$ prediction performance using diffuse reflectance spectra data

\begin{tabular}{lllll}
\hline Spectrum & \multicolumn{4}{l}{ Statistical indicator } \\
& $\mathrm{R}^{2}$ & $\mathrm{r}$ & RMSE & RPD \\
\hline \hline Raw & 0.75 & 0.87 & 0.26 & 1.73 \\
DT & 0.80 & 0.90 & 0.21 & 2.70 \\
\hline
\end{tabular}

$R^{2}$ : coefficient of determination, $r$ : correlation coefficient, RMSE: root means square error, $R P D$ : residual predictive deviation, DT: de-trending.
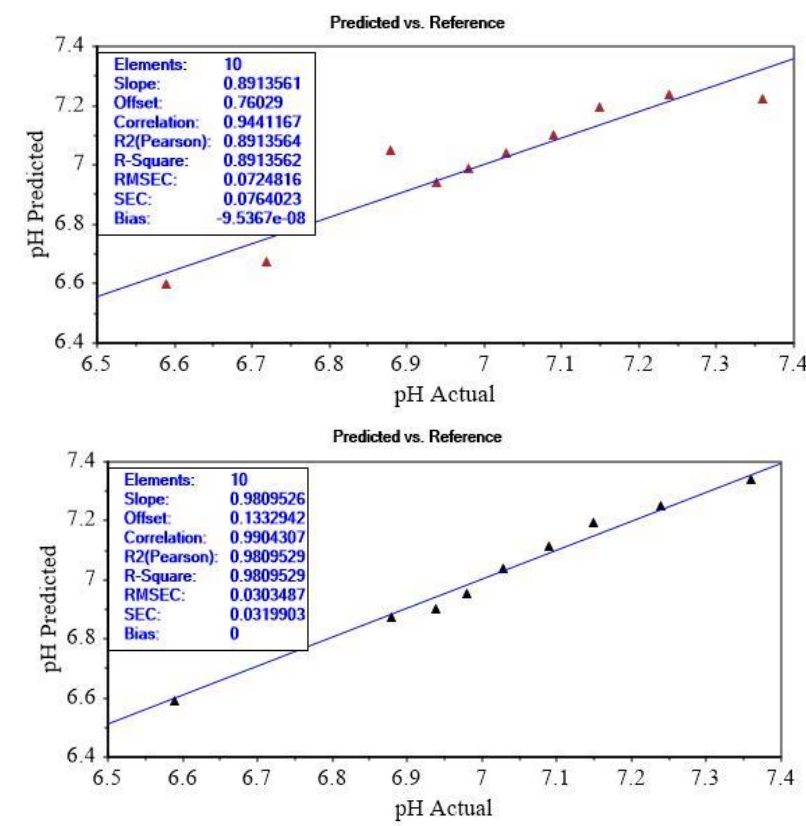

Fig. 4. Scatter plot between actual and predicted $\mathrm{pH}$ of soil samples using NIR spectra data.

Judging from obtained prediction results, both soil quality indices in form of C-organic and $\mathrm{pH}$, we may argue that NIRS can be employed and applied as an alternative fast, effective and non-destructive method to determine and predict soil quality. We may also use this particular method to predict other soil quality indices with the satisfactory result as well as previous studies. Further research, of course, is still required to develop prediction models and validate using independent data. 


\section{Conclusion}

Based on obtained results, we may conclude that near-infrared spectroscopy can be applied as a rapid alternative method used to predict soil organic carbon (SOC) and $\mathrm{pH}$. Spectra correction using De-trending (DT) was obviously improved prediction accuracy and robustness by increasing correlation coefficient between actual and predicted $\mathrm{C}$-organic and $\mathrm{pH}$, increasing RPD index as well as lowering RMSE error prediction. DT spectra correction provide the most accurate and robust $\mathrm{C}$-organic prediction result with correlation coefficient $r=0.94$ and $\mathrm{RPD}=$ 3.96. whilst for $\mathrm{pH}$ prediction, correlation coefficient $\mathrm{r}=0.99$ and RPD index $=4.84$ respectively.

\section{Acknowledgments}

We sincerely acknowledge to LPPM Universitas Syiah Kuala for providing research grant and funding through Hibah Penelitian Professor Scheme 2018.

\section{REFERENCES}

[1] M. Knadel et al., "Comparing predictive ability of laser-induced breakdown spectroscopy to visible near-infrared spectroscopy for soil property determination," Biosyst. Eng., vol. 156, pp. 157-172, 2017.

[2] A. M. Mouazen, B. Kuang, J. de Baerdemaeker, and H. Ramon, "Comparison among principal component, partial least squares and back propagation neural network analyses for accuracy of measurement of selected soil properties with visible and near infrared spectroscopy.," Geoderma, vol. 158, no. 1/2, pp. 23-31, 2010.

[3] J. Moros et al., "Use of Reflectance Infrared Spectroscopy for Monitoring the Metal Content of the Estuarine Sediments of the Nerbioi-Ibaizabal River (Metropolitan Bilbao, Bay of Biscay, Basque Country)," Environ. Sci. Technol., vol. 43, no. 24, pp. 93149320, Dec. 2009.

[4] H.-Y. Ren, D.-F. Zhuang, A. N. Singh, J.-J. Pan, D.-S. Qiu, and R.-H. Shi, "Estimation of As and $\mathrm{Cu}$ Contamination in Agricultural Soils Around a Mining Area by Reflectance Spectroscopy: A Case Study,” Pedosphere, vol. 19, no. 6, pp. 719-726, 2009.

[5] A. A. Munawar, D. von Hörsten, J. K. Wegener, E. Pawelzik, and D. Mörlein, "Rapid and non-destructive prediction of mango quality attributes using Fourier transform near infrared spectroscopy and chemometrics," Eng. Agric. Environ. Food, vol. 9, no. 3, pp. 208-215, 2016.

[6] T. Shi, Y. Chen, Y. Liu, and G. Wu, "Visible and near-infrared reflectance spectroscopy-An alternative for monitoring soil contamination by heavy metals," $J$. Hazard. Mater., vol. 265, pp. 166-176, 2014.

[7] M. J. Salazar, J. H. Rodriguez, G. L. Nieto, and M. L. Pignata, "Effects of heavy metal concentrations $(\mathrm{Cd}, \mathrm{Zn}$ and $\mathrm{Pb})$ in agricultural soils near different emission sources on quality, accumulation and food safety in soybean [Glycine max (L.) Merrill]," J. Hazard. Mater., vol. 233-234, pp. 244-253, 2012.

[8] Q. Li et al., "Health risk of heavy metals in food crops grown on reclaimed tidal flat soil in the Pearl River Estuary, China," J. Hazard. Mater., vol. 227-228, pp. 148-154, 2012.

[9] J. Wang, L. Cui, W. Gao, T. Shi, Y. Chen, and Y. Gao, "Prediction of low heavy metal concentrations in agricultural soils using visible and near-infrared reflectance 
spectroscopy," Geoderma, vol. 216, pp. 1-9, 2014.

[10] G. S. Senesi and N. Senesi, "Laser-induced breakdown spectroscopy (LIBS) to measure quantitatively soil carbon with emphasis on soil organic carbon. A review," Anal. Chim. Acta, vol. 938, pp. 7-17, 2016. 